\title{
Anomalous transport of energetic particles in ITER relevant scenarios
}

\author{
M. Albergante, ${ }^{1, a)}$ J. P. Graves, ${ }^{1}$ A. Fasoli, ${ }^{1}$ F. Jenko, ${ }^{2}$ and T. Dannert ${ }^{1}$ \\ ${ }^{1}$ Centre de Recherches en Physique des Plasmas, Association EURATOM-Confédération Suisse, \\ 1015 Lausanne, Switzerland \\ ${ }^{2}$ Max-Planck-Institut für Plasmaphysik, Boltzmannstrasse 2, D-85748 Garching, Germany
}

(Received 26 June 2009; accepted 12 October 2009; published online 2 November 2009)

\begin{abstract}
The anomalous transport of energetic ions in the presence of turbulent fields is investigated. Nonlinear simulations of a steady-state ITER [R. Aymar et al., Nucl. Fusion 41, 1301 (2001)] scenario have been carried out using the gyrokinetic turbulence code GENE [F. Jenko et al., Phys. Plasmas 7, 1904 (2000)], modeling the suprathermal particles as high temperature Maxwellian distributions in the passive tracer limit. Velocity space analysis shows that single particle diffusivities of ions above the critical energy are significantly larger than their neoclassical counterpart. () 2009 American Institute of Physics. [doi:10.1063/1.3257913]
\end{abstract}

\section{INTRODUCTION}

The confinement of suprathermal ions in fusion reactors is of primary importance for creating and sustaining burning plasma conditions. First, energetic particles must transfer their energy to the background species before being transported out of the plasma. Second, the ejection of even a small fraction of suprathermal particles can cause a significant heat load on plasma facing materials, posing a serious threat to long term operation. Several classes of high energy ions will characterize ITER, ${ }^{1}$ the first tokamak capable of sustaining steady-state discharges characterized by a significant value of Q, the ratio of fusion power, and power input to the machine. Suprathermal particles in ITER can be created by fusion reactions ( $\alpha$ particles), neutral beam injection (NBI), or ion cyclotron radio frequency heating (ICRH). NBI distributions are mainly constituted by passing particles, while a large fraction of ICRH heated ions can be modeled as deeply trapped. Alpha particle distributions are generally isotropic and contain both kinds of particles. The main difference between trapped and passing ions is the pitch angle, the angle between the particle velocity vector and the local magnetic field. This quantity is strictly related to the orbital motion and the Larmor radius of a charged particle in a tokamak. It also plays an important role in determining the interaction between energetic ions and plasma waves. Whenever this interaction is significant, the transport of energetic ions can become important. Detailed analyses are then needed to estimate how effectively fast particles can transfer their energy to the background plasma before being expelled.

Among the several anomalous transport mechanisms, Alfvén mode interactions with fusion born particles and magnetic ripple losses of energetic ions have received a great deal of attention over many years. An overview of these transport mechanisms and estimates for ITER can be found in Ref. 2. In contrast, the transport of suprathermal particles driven by microturbulence has not been a subject of concentrated research, especially following the work of a number of groups concluding that fast gyroaveraging and orbit averag-

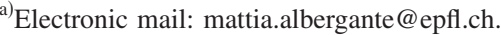

ing of the turbulent fields would render the transport negligible. ${ }^{3-5}$ An apparent confirmation of these theoretical conclusions was found on early experiments at the Tokamak Fusion Test Reactor (TFTR) ${ }^{6-9}$ where an upper limit for fast ion diffusivities was found at $0.1 \mathrm{~m}^{2} \mathrm{~s}^{-1}$, an order of magnitude smaller than that of thermal ions. Recently, however, there has been renewed interest, triggered by experimental observations of anomalous redistribution of NBI ions at the Axial Symmetric Divertor EXperiment (ASDEX) Upgrade, ${ }^{10,11}$ at the Joint European Torus (JET),${ }^{12,13}$ at the Mega-Ampere Spherical Tokamak (MAST), ${ }^{14,15}$ and at DIII-D. ${ }^{16,17}$

On the theoretical side, earlier results on the transport of particles characterized by large energies have recently been extended. First-principles calculations of the transport of trace ions in a slab geometry indicated that the complex interplay of gyroaveraging, field anisotropies, and poloidal drift effects can lead to non-negligible diffusivities for energetic particles. ${ }^{18,19}$ Flux tube simulations by means of the GYRO code ${ }^{20}$ showed how large fluxes could characterize the transport of high temperature populations in the passive tracer (i.e., not backreacting onto the turbulent fields) limit. ${ }^{21}$ According to the conclusions of Refs. 3-5, it would be expected that increasing the number of high energy particles, by raising the temperature of a passive population, would reduce the particle transport due to gyro- and orbit-averaging effects. This, however, did not occur in Ref. 21, as fluxes four times larger than the background ion flux were observed for $T_{\alpha}=40 T_{e}$. Nevertheless, Angioni et al. ${ }^{22}$ claimed that the conclusions drawn in Ref. 21 could be affected by the particular normalization chosen for the quantities studied. In Refs. 22 and 23, linear and nonlinear simulations by means of three different gyrokinetic codes [GYRO, GS2 $2{ }^{24,25}$ and GKW (Ref. 26)] have been carried out, showing that the transport of a slowing down distribution is negligible in ITER.

The analysis of Refs. 21-23, although rigorous from a numerical point of view, drew conclusions based on ensemble transport properties from the entire distribution function, rather than considering the contribution to the overall transport given by particular regions in phase space. The extension to a velocity space resolved analysis by means of 
the GENE $\operatorname{code}^{27,28}$ was undertaken in Ref. 29 , where a $1 / E$ decay of the particle diffusivity of high energy passing particles was observed in the presence of small scale turbulence. This scaling shows how microturbulence can affect NBI ions, whose energies for present day experiments can be 10-20 times larger than the electron temperature. In particular, the conclusion that turbulent transport could be responsible for the anomalous redistribution of NBI driven current observed on ASDEX Upgrade ${ }^{11}$ was drawn. The inclusion of trapped ions and a more detailed velocity space analysis were performed by means of the GTC $\operatorname{code}^{30}$ using the particle-in-cell technique. The latter paper confirmed the energy dependence for passing particles, but obtained a fast energy decay for the trapped population. Both of these dependencies were explained in terms of combined gyroaveraging and guiding center drift orbit averaging, the latter characterizing both classes of particles while the former only trapped ions. A single particle approach was employed in Ref. 31, where the same dependence for passing ions already observed in the literature was recovered. At the same time, a different high energy decay for trapped particles was discovered. Moreover, the physical interpretation of the transport mechanism was different, as it was demonstrated that orbit averaging is no longer valid for particles whose energies largely exceed the plasma temperature. The faster energy decay for trapped ions was due to gyroaveraging, whose effectiveness was not questioned.

In the present work, we study the anomalous transport of energetic ions in ITER by modeling both the distribution of energetic particles and the background plasma profiles that create the turbulent fields. We develop our study by first surveying the several quantities and relevant normalizations found in the literature. The analysis of the transport of passive tracers in a simple case, employing a normalization allowing for a qualitative investigation, is then presented. This exercise identifies the main parameters influencing the transport of Maxwellian distributions, as well as the transport features to investigate more accurately when simulating ITER discharges. In our case, among the relevant phenomena are an impurity pinch for thermal distributions and a nonvanishing outward diffusivity characterizing high temperature populations. This phenomenon, in particular, is counterintuitive considering the expected beneficial effects of field gyroaveraging. We therefore extend the analysis by modeling an ITER steady-state scenario discharge in the $s$ - $\alpha$ geometry, an equilibrium model with nested circular flux surfaces. For modeling the ITER discharge, we employ the temperature and density profiles for the background species calculated with the ASTRA transport code. We shall then introduce a simple model for obtaining the ash impurity and alpha particle population profiles. We show how the low temperature pinch of helium ash disappears, but still a non-negligible diffusivity characterizes high temperature distributions. To deduce which particles of a Maxwellian distribution are mostly affected by turbulent transport, we analytically introduce a velocity space dependent diffusivity. Using this definition, we show how high energy particles can be transported above neoclassical expectations. Finally, the differences with respect to a purely electrostatic case are presented, showing that finite $\beta$ effects must be retained in gyrokinetic simulations of burning plasmas.

The paper is organized as follows. In Sec. II, the model describing the mathematical and numerical approach is outlined. In particular, the GENE code and its main features are introduced, as well as the transport quantities that are employed throughout the paper. In Sec. III, an ITER discharge is analyzed and modeled. The transport of suprathermal populations in the Maxwellian limit is studied in detail. The transport features of small regions of velocity space are analyzed in Sec. IV. This approach allows us to go beyond the Maxwellian limit and focus on single particle diffusivities. Comparisons to simulations at low $\beta$ are presented in Sec. V. Finally, the results are summarized in Sec. VI.

\section{THE MODEL}

The simulations are performed by means of the gyrokinetic turbulence code GENE with circular flux surface equilibria in the $s$ - $\alpha$ geometry. ${ }^{32}$ The code exploits Eulerian techniques for solving the gyrokinetic Vlasov equation assuming Maxwellian stationary distributions $\left(f_{0}\right)$. The fivedimensional simulation space is described by the set of coordinates $\left(x, y, z, v_{\|}, \mu\right)$, where $x$ is a radial coordinate and $y$ and $z$ are nonorthogonal coordinates lying on the flux surfaces. Finally, $\left(v_{\|}, \mu\right)$ are the velocity space variables, where $\mu=m v_{\perp}^{2} /(2 B)$. GENE is able to work with an arbitrary number of particle species, either active or passive. The latter are assumed to carry negligible modifications to the structure of the turbulent fields in a real plasma, which is a good approximation for low concentration of energetic ions ${ }^{22}$ in an actual discharge.

A Maxwellian model is used to examine the levels of anomalous transport of energetic particles. Temperature scans are performed to evaluate gyro- and orbit-averaging effects on the particle transport. A key quantity is the time average over the saturated phase of the electrostatic component of the nonlinear particle flux $\mathbf{\Gamma}=\int d \mathbf{v} \delta f \delta \mathbf{v}$,

$$
\boldsymbol{\Gamma}^{e s}=\int d \mathbf{v} \delta f \delta \mathbf{v}_{E \times B},
$$

where

$$
\delta \mathbf{v}_{E \times B}=-\frac{\nabla \delta \Phi \times \mathbf{B}}{B^{2}} .
$$

Here, the perturbed electrostatic field has been averaged over the fast gyromotion of the charged particles. We caution the reader that the contribution that we refer to as electrostatic does of course retain electromagnetic effects in $\delta f$ via the electromagnetic fields. The nomenclature comes from the choice of exclusively the $E \times B$ component of the perturbed velocity in the integral of Eq. (1). The employed magnetic contributions contained in the perturbed distribution function $\delta f$ become important for finite $\beta$ plasmas and, as we shall see in Sec. V, must be retained in the simulations. As gyroaveraging reduces the amplitude of the turbulent fields and consequently of the turbulent flux by a factor $J_{0}\left(k_{\perp} v_{\perp i} / \Omega_{c i}\right)$, only modest transport is expected for distributions with large energy content. From a simple linear analysis it can be 
shown that $\delta f \propto \nabla f_{0}$. The gradient of a Maxwellian distribution $f_{0}$ can be analytically calculated from

$$
f_{0}(E, \mathbf{x})=\frac{n(\mathbf{x})}{T(\mathbf{x})^{3 / 2}} e^{-E / T(\mathbf{x})},
$$

such that

$$
\boldsymbol{\nabla} f_{0}=f_{0}[\boldsymbol{\nabla} \ln n+(E / T-3 / 2) \boldsymbol{\nabla} \ln T] .
$$

The particle flux of Eq. (1) can be thus decomposed in two terms,

$$
\boldsymbol{\Gamma}=\int d \mathbf{v} \delta f \delta \mathbf{v}_{E \times B} \simeq A \boldsymbol{\nabla} \ln n+A^{\prime} \boldsymbol{\nabla} \ln T .
$$

$A$ and $A^{\prime}$ are the parameters responsible for the anomalous transport of the population. The $\nabla \ln T$ term contained in the equation is usually negative and gives rise to a countergradient flux (typically radially inward). On the other hand, the $\boldsymbol{\nabla} \ln n$ term drives an outward flux that nonlinearly reduces the density gradient. These relations will be particularly useful later in the paper. As we are mostly interested in particle fluxes and diffusivities along the $x$-direction, we will drop the bold character notation whenever considering projections over the radial coordinate of the vectorial quantities studied. As we will be focusing only on $\boldsymbol{\Gamma}^{e s}$ of Eq. (1), we will also drop the es superscript.

\section{A. Definition of transport quantities and normalizations}

Throughout the papers cited in Sec. I, the study of the transport of energetic ions has focused on several different quantities. We hereby report a list of the different normalizations of the particle flux defined in Eq. (1):

- Flux per particle $(\alpha)$ scaled to background ion $(i)$ flux per particle. This normalization, mainly applied in Ref. 21, is based on the definition of a flux per particle as $\Gamma_{j} / n_{j}$, with $\Gamma$ given, e.g., by Eq. (1). The ratio of this variable to the background ion flux per particle is therefore

$$
\hat{\Gamma}_{\alpha}=\left(\Gamma_{\alpha} / n_{\alpha}\right) /\left(\left|\Gamma_{i}\right| / n_{i}\right) \text {. }
$$

The results can be misleading, however, when alpha particles and background thermal ions are characterized by different temperature and density profiles. The electrostatic particle flux $\Gamma$ is proportional to both $\nabla n$ and $\nabla T$ of the population, as described in Eq. (5). When normalizing the flux of two populations characterized by different gradients, the results should then be considered with caution, especially when the background ion flux is small. ${ }^{22}$

- Effective particle diffusivity scaled to ion values. In this case, the analysis relies on the definition of an effective diffusivity for the $j$ th species as $D_{j}^{\text {eff }}=-\Gamma_{j} / \nabla n_{j}$. The alpha particle diffusivity can be quantified in relation to the background ion diffusivity

$$
\hat{D}_{\alpha}^{\mathrm{eff}}\left(T_{\alpha}\right)=D_{\alpha}^{\mathrm{eff}}\left(T_{\alpha}\right) /\left|D_{i}^{\mathrm{eff}}\right| .
$$

The density gradient dependence of the particle flux is then partially removed. When $\Gamma_{i}$ is negligible, however, the same limitations described for Eq. (6) still hold.

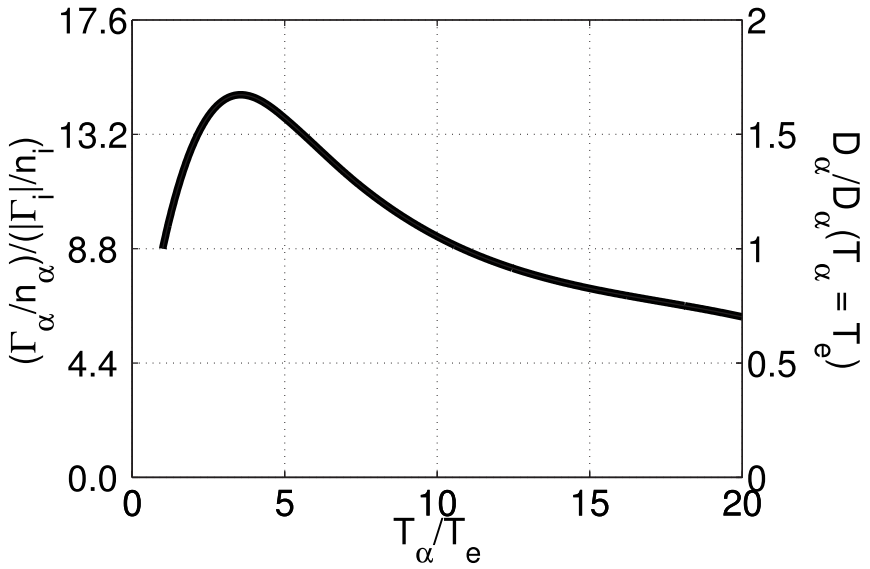

FIG. 1. Flux per particle normalization (left axis) compared with an effective diffusivity normalization (right axis) as a function of the temperature of the population considered.

- Diffusivity scaled to thermal values. The effective diffusivity of a suprathermal population is normalized to the $D^{\text {eff }}$ of the same population as if it were in thermal equilibrium with the bulk plasma

$\hat{D}_{\alpha}^{\mathrm{eff}}\left(T_{\alpha}\right)=D_{\alpha}^{\mathrm{eff}}\left(T_{\alpha}\right) /\left|D_{\alpha}^{\mathrm{eff}}\left(T_{\alpha}=T_{e}\right)\right|$.

This choice, mainly adopted in Ref. 22 and in the present work, is particularly useful when the analysis is focused on the estimate of gyro- and orbit-averaging effects on the transport of passive species. From now on, we will drop the "eff" superscript from the diffusivity variable to make the notation clearer.

In Fig. 1 we can see how two different normalizations rescale an otherwise identical curve, and consequently different conclusions might be drawn if the two curves are viewed independently. We plot $\hat{\Gamma}_{\alpha}$ according to Eq. (6) as a function of the temperature of the passive Maxwellian. At the same time, we put $\hat{D}_{\alpha}$ of Eq. (8) on the right axis of the same simulation. The simulation is characterized by the following parameters: $q_{0}=1.4, \hat{s}=0.8$, a radial box of $50 \rho_{s},(x, y, z)$ $=(128,32,16)$ points in real space, $\left(v_{\|}, \mu\right)=(32,8)$ in velocity space, and a reduced mass ratio $m_{i} / m_{e}=400$. Defining

$$
\begin{aligned}
& \Omega_{n}=-\frac{R_{0}}{n} \frac{d n}{d r}=R_{0} / L_{n}, \\
& \Omega_{T}=-\frac{R_{0}}{T} \frac{d T}{d r}=R_{0} / L_{T},
\end{aligned}
$$

the background species are characterized by $\Omega_{n_{e, i}}=3, \Omega_{T_{e, i}}$ =9. For the passive population we have chosen $m_{\alpha}=2 m_{i}$, $\Omega_{n_{\alpha}}=3$, and $\Omega_{T_{\alpha}}=0$. By considering a flux per particle normalization $\left(\hat{\Gamma}_{\alpha}\right)$ as given by Eq. (6) and plotted on the left axis of Fig. 1, one may conclude that in a tokamak suprathermal populations with $T_{\alpha}=20 T_{e}$ are transported across the flux surfaces as much as six to seven times faster than the background species, and that field averaging effects are not sufficient to suppress the particle transport. The alpha and background ion species, however, are characterized by different temperature gradients. As $\nabla T_{\alpha}=0$ for the passive 


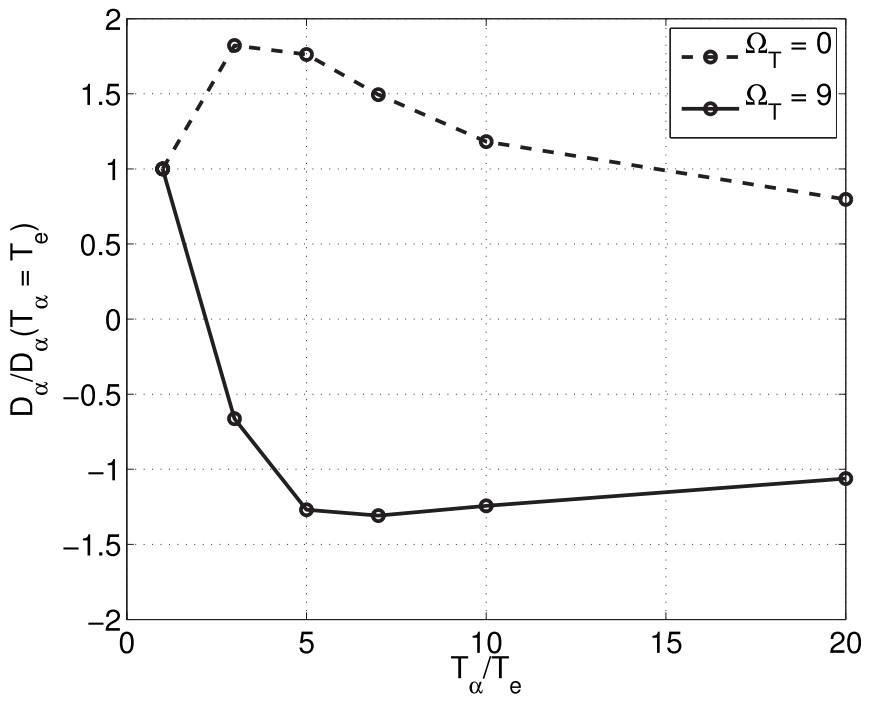

FIG. 2. Effective diffusivity of a Maxwellian distribution as a function of temperature for two different populations: ash $\left(\Omega_{T}=9\right)$ and alphas $\left(\Omega_{T}=0\right)$. High temperature populations exhibit either thermodiffusive characteristics for peaked temperature gradients (ash) or large diffusivities for flat temperature distributions (alphas).

population, the $\nabla n$-driven diffusion is not balanced by any thermodiffusive pinch. These tracers are therefore expected to diffuse faster than the ions, given that $\Omega_{T_{i}}=9$. The observation of a higher flux is then related to the choice of the profile gradients and similar misleading conclusions would be drawn if one chooses to study the transport in terms of $D_{\alpha} /\left|D_{i}\right|$ from Eq. (7). When dealing with the $\hat{D}_{\alpha}$ diffusivity from Eq. (8), the interpretation differs significantly. As their kinetic energy becomes bigger, the fast ions will tend to feel weaker fields due to field averaging effects. We can conclude that the transport of the alpha population is reduced by $20 \%$ with respect to a thermal case when the temperature of the alpha distribution is 20 times larger than the plasma temperature. We can now see how a significant ratio is present between the definition of Eqs. (6) and (8) for the case considered in Fig. 1. From now on, we analyze the transport as a function of the effective diffusivity defined in Eq. (8), since we are mostly interested in the gyro- and orbit-averaging effects on the transport. Absolute values are considered only when the analysis is focused on the particle diffusivity of ITER fast ions in a specific reference scenario.

Let us now consider the results of the simulation shown in Fig. 2. Here, the same parameters previously introduced for the bulk plasma have been employed. The passive species are characterized by $\Omega_{n}=3$, while a separation has been introduced in the temperature of high and low energy ${ }^{4} \mathrm{He}$ populations, namely, $\Omega_{T}^{\alpha}=0$ and $\Omega_{T}^{\text {ash }}=9$. The flat temperature profile is more representative of alpha particles, whose high energies $\left(E_{\alpha} \geqslant 3.5 \mathrm{MeV}\right)$ are not significantly modified by the properties of the thermal plasma. The choice of a peaked temperature profile has been made to model helium impurities ("ash"). These particles represent thermalized fusion products and typically inherit the characteristics of the bulk plasma. These parameters are similar to those of Ref. 21. The results presented in this figure suggest that an inward

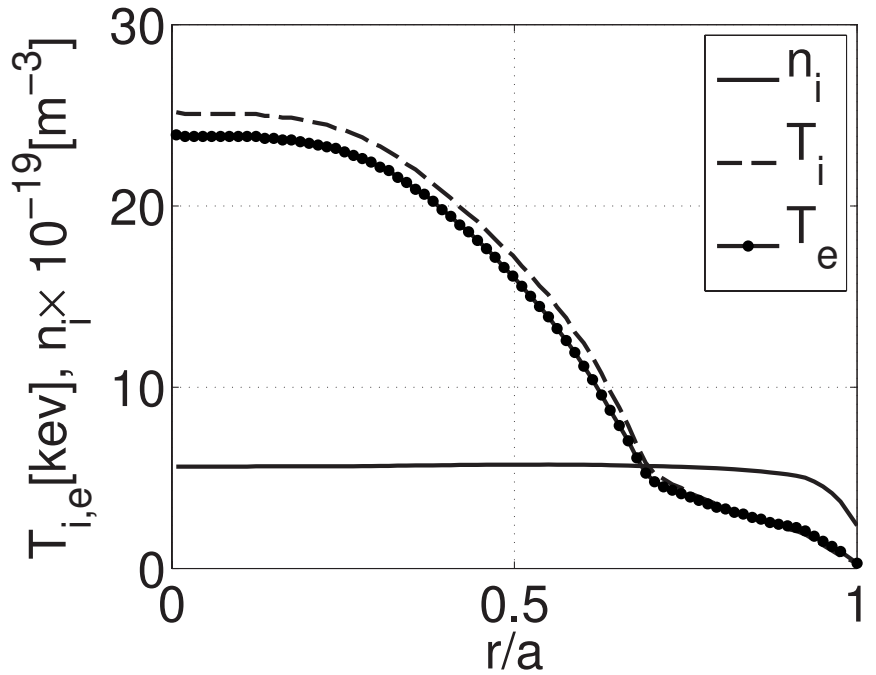

FIG. 3. Density and temperature profiles of the bulk plasma for an ITER steady-state scenario discharge. These profiles have been used for determining the characteristics of the alpha particle ITER populations.

particle flux, due to thermodiffusive effects, will arise for the $\Omega_{T}^{\text {ash }}=9$ ash distribution when its temperature exceeds roughly two times the background ion temperature. This undesirable situation would lead to an accumulation of impurities in the core, thus decreasing the fusion production rate and enhancing bremsstrahlung losses. The flat temperature distribution $\Omega_{T}^{\alpha}=0$ shows an outward particle flux for all temperatures considered. The intensity of the flux will not vanish by increasing the temperature, and a decrease of only $20 \%$ in $D_{\alpha}$ is observed when $T_{\alpha}=20 T_{e}$. Two questions arise at this point. First, is it possible that suprathermal ash will be pinched toward the core of an actual fusion device? Second, is the high temperature particle diffusivity a reliable estimate for fusion born particles in future tokamaks? To answer these two questions, thermal density and temperature profiles need to be carefully modeled.

\section{ITER MODELING}

In this section, our simulations focus on an ITER steadystate scenario discharge, ${ }^{33,34}$ designed for long pulses with $Q>5$, where $Q$ is the ratio of fusion power and power input to the plasma.

The profiles in Fig. 3 have been employed to define the background plasma parameters at midradius, $\Omega_{n_{i, e}}=0.5, \Omega_{T_{i}}$ $=7, \Omega_{T_{e}}=0$. A flat temperature profile is chosen to focus on ion temperature gradient (ITG) modes. The magnetic field configuration has been modeled by choosing $q_{0}=1.5$ and a low shear $\hat{s}=0.4$. An artificially low ion to electron mass ratio $\left(m_{i} / m_{e}=400\right)$ has been employed to save computational time while retaining the most important properties of the instability considered. These simulations are characterized by a radial extension of $L_{x}=200 \rho_{s}$ and by the following grid parameters: $N_{x}=128, N_{k_{y}}=32, k_{y}^{\min } \rho_{s}=0.05, N_{z}=32, N_{v_{\|}}=32$, and $N_{\mu}=8$, where $N$ is the number of points in a particular direction and $k$ is the wave number of the perturbation. We have chosen $\beta_{\text {midradius }}=0.6 \%$, a value safely below the threshold for the onset of kinetic ballooning modes (KBM, 
Ref. 35), expected in this case at $\beta_{c}=1.2 \%$. We again employ $s$ - $\alpha$ geometry with $\alpha$ set to zero. A comparison between the linear phase of two simulations, one with $\alpha=0$ and the other employing the actual value of $\alpha$, has been carried out, showing that no modifications to the mode structure are present due to the effect of finite $\beta$ on the magnetic geometry. An ITG instability is dominant with the most unstable mode peaking at $k_{y} \rho_{s}=0.3$.

An isotropic slowing down distribution is taken to model the velocity distribution of the fast particles subject to classical collisions

$$
f_{s}(r, v)=\frac{S_{0}(r) \tau_{s}(r)}{4 \pi} \frac{\Theta\left(v_{\alpha}-v\right)}{v^{3}+v_{c}^{3}} .
$$

Here $S_{0}$ is the fusion production rate, $\tau_{s} \propto T_{e}^{3 / 2}(r) / n_{e}(r)$, and $v_{c}=\left[3 / 4 \sqrt{\pi} m_{e} / m_{\alpha} Z_{\mathrm{eff}}(r)\right]^{1 / 3} v_{\text {the }}(r)$. The GENE code works with Maxwellian stationary distributions, and consequently some approximations need to be made. Following the analytical treatment of Refs. 21 and 22, we model the alpha particle distribution function as a Maxwellian distribution characterized by "equivalent" density and temperature profiles. The equivalent density is given by the zeroth moment in velocity space of the slowing down distribution function. The temperature $T_{\alpha}(r)$ of the equivalent Maxwellian is equal to the second moment of the alpha particle distribution function. The key point of this procedure is replacing the slowing down function with a Maxwellian distribution characterized by the same pressure,

$$
\begin{aligned}
& n_{\alpha}(r)=\int f_{s}(r, v) d \mathbf{v}, \\
& T_{\alpha}(r)=\frac{m_{\alpha}}{n_{\alpha}} \int v^{2} f_{s}(r, v) d \mathbf{v} .
\end{aligned}
$$

These integrals are calculated while separating between low and high energy ranges in velocity space. Specifically, the equivalent density profile of thermal (ash) particles is obtained by restricting the integral of Eq. (10) to thermal velocities. The density profile of energetic ("hot") particles, on the other hand, is provided by integrating over a suprathermal energy range. The same treatment is applied to the calculation of the equivalent temperature of the Maxwellian

$$
\begin{aligned}
& n_{\alpha}^{\text {hot }}(r)=\int_{2 v_{\mathrm{th}_{\mathrm{i}}}}^{v_{\alpha}} f_{s}(r, v) d \mathbf{v}, \\
& n_{\alpha}^{\text {ash }}(r)=\int_{0}^{2 v_{\mathrm{th}_{\mathrm{i}}}} f_{s}(r, v) d \mathbf{v}, \\
& T_{\alpha}^{\mathrm{hot}}(r)=\frac{m_{\alpha}}{n_{\alpha}^{\text {hot }}(r)} \int_{2 v_{\mathrm{th}_{\mathrm{i}}}}^{v_{\alpha}} v^{2} f_{s}(r, v) d \mathbf{v}, \\
& T_{\alpha}^{\text {ash }}(r)=\frac{m_{\alpha}}{n_{\alpha}^{\text {ash }}(r)} \int_{0}^{2 v_{\mathrm{th}_{\mathrm{i}}}} v^{2} f_{s}(r, v) d \mathbf{v} .
\end{aligned}
$$

Here $v_{\alpha}$ is the birth velocity of the alpha particle. The resulting profiles are shown in Fig. 4. By taking the radial deriva-
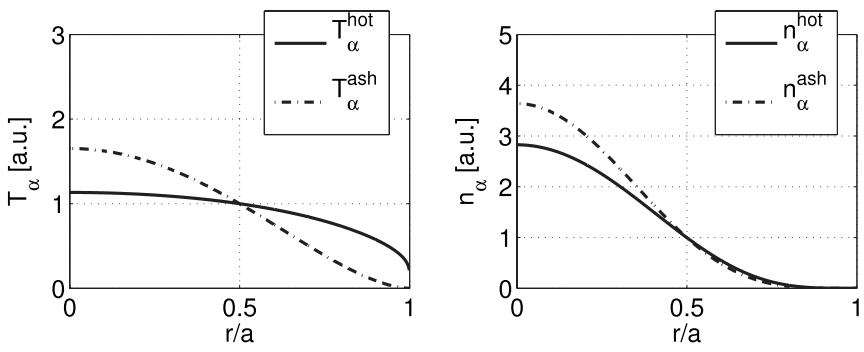

FIG. 4. ITER alpha particle profiles, normalized at midradius, as obtained from integration of the slowing down distribution function of alpha particles. A separation between thermal (ash) and suprathermal (hot) particles has been introduced in the integration.

tive of these profiles we obtain the parameters for the GENE simulation $\left(\Omega_{n_{\alpha}}^{\text {hot/ash }}=15 / 18, \Omega_{T_{\alpha}}^{\text {hot/ash }}=1.5 / 7\right.$ at $r / a=0.5$ for species with $\left.m_{\alpha}=2 m_{i}\right)$. For each species, again we perform a temperature scan, as shown in Fig. 5, to see whether the low temperature pinch appears for the ash. In addition, we want to see how effectively is the transport of the high energy population suppressed by field averaging effects.

From Fig. 5, where the diffusion coefficient is expressed in absolute units, it is clear that the inward flux for the ash is absent, mainly due to the presence of strong density gradients overcoming the thermodiffusive pinch. Due to the possible drawbacks of impurity retention, this result represents a piece of good news for ITER operation. Considering the high energy particles, diffusivities above $0.4 \mathrm{~m}^{2} \mathrm{~s}^{-1}$ are observed over the temperature range $T_{\mathrm{hot}}=20-50 T_{e}$. The evidence of a non-negligible transport of thermal distributions with a large energy content points to the likelihood of having an enhancement in the diffusivity of energetic ions in ITER. Nevertheless, some clarifications should be made at this point. First, evaluating the transport of an entire distribution clearly gives only an average picture and does not exclude that the enhanced diffusivity is due to the lower energy particles only. Second, employing thermal or slowing down distributions is only valid as long as collisional processes constitute the dominant transport channel. ${ }^{36}$ If anomalous transport becomes significant, the assumption of collisional slowing down might be inaccurate. To overcome these difficulties and draw conclusions on the microturbulence driven transport of fast ions, we now focus on velocity space resolved analysis.
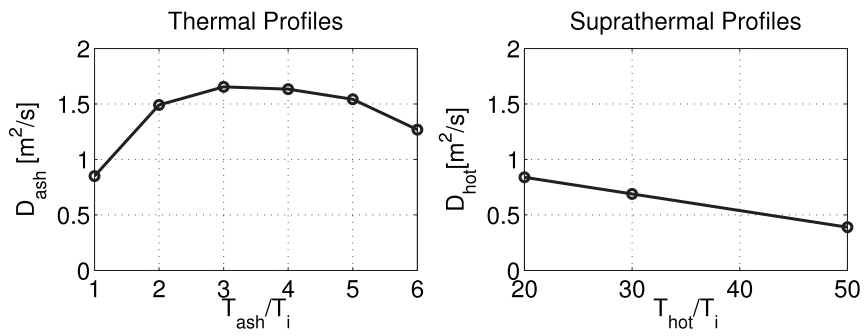

FIG. 5. Particle diffusivity of Maxwellian populations in ITER modeled according to the profiles of Fig. 4. For low temperature distributions the pinch present in Fig. 2 is not observable. A residual diffusivity at high temperatures is still present, thus indicating the possibility of an enhanced transport of alpha particles in ITER. 


\section{VELOCITY SPACE INVESTIGATION}

Within the same simulation we have just analyzed, we focus our attention on the population with temperature $T_{\alpha}$ $=30 T_{e}$. In the passive tracer limit, we introduce a set of variables that allow us to resolve the particle diffusivity variable in velocity space.

To define a kinetic description of the diffusivity that is analogous to the fluid particle diffusivity, we start by defining a microscopic particle flux per unit of velocity space volume $\Gamma_{v}$, such that $\Gamma \equiv \int d \mathbf{v} \Gamma_{v}$. From Eq. (1) it follows that

$$
\Gamma_{v}=\delta f \delta v_{E \times B} .
$$

The velocity space dependent particle diffusivity $D_{v}$ is defined in terms of $\Gamma_{v}$ and $\nabla f_{0}$ as

$$
D_{v}=-\frac{\delta f}{\nabla f_{0}} \delta v_{E \times B}=-\frac{\Gamma_{v}}{\nabla f_{0}} .
$$

This equation can be considered as a generalized Fick's law, where $D_{v}$ has units of $\mathrm{m}^{2} \mathrm{~s}^{-1}$, like its fluid counterpart. In the simulations presented in Sec. III, the approximation $\nabla \ln T$ $\ll \nabla \ln n$ holds both for ash and energetic ion distributions. Equation (4) can then be rewritten as $\nabla \ln f_{0}=\nabla \ln n$, which simplifies the expression for $D_{v}$,

$$
D_{v}=-\frac{1}{\nabla \ln n} \frac{\delta f \delta v_{E \times B}}{f_{0}} .
$$

Fick's law is satisfied by the velocity space average of $D_{v}$ over the equilibrium distribution function

$$
D=\frac{\int d \mathbf{v} f_{0} D_{v}\left(v_{\|}, \mu\right)}{\int d \mathbf{v} f_{0}} .
$$

The variable $\delta f \delta v_{E \times B}$ is provided by the velocity space diagnostic in GENE as a function of the parallel coordinate $z$ and of the time. We define the following averages of an arbitrary function $\chi$ :

$$
\langle\chi\rangle_{z}=\frac{\int_{-\pi}^{\pi} d z J(z) \chi}{\int_{-\pi}^{\pi} d z J(z)} \quad \text { and } \quad\langle\chi\rangle_{t}=\frac{1}{\Delta t} \int_{t_{\min }}^{t_{\max }} \chi d t,
$$

where $J$ is the Jacobian in $z$. The phase space resolved diffusion coefficient employed in the following analysis is then

$$
D_{v}\left(v_{\|}, \mu\right)=\left\langle\left\langle D_{v}\left(v_{\|}, \mu, z, t\right)\right\rangle_{z}\right\rangle_{t} .
$$

In order to facilitate the interpretation of the results, the value of $0.1 \mathrm{~m}^{2} \mathrm{~s}^{-1}$ is assumed as the discriminant between low and high diffusivity regimes, as it represents an upper limit of the neoclassical diffusivity of fast ions. ${ }^{37}$ We will consider transport to be enhanced when a particle exhibits a radial diffusion larger than $0.1 \mathrm{~m}^{2} \mathrm{~s}^{-1}$ at energies comparable or larger than $E_{c}$. Below this value, energetic particles have already given most of their energy to the background electrons and pitch angle scattering will enhance collisional diffusivity.

Velocity dependent values of the radial diffusion coefficient are shown in Fig. 6. We can see that diffusivities larger than $D_{\text {neo }}$ are observed over a wide range of energies, indicating that not only thermal particles are significantly transported by microturbulence. To compare these results with the

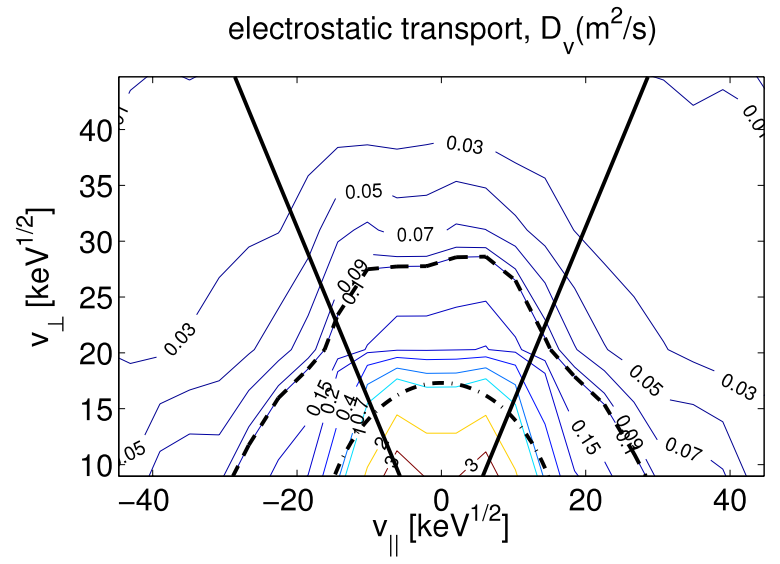

FIG. 6. (Color online) Anomalous transport of a passive species in ITER as a function of the velocity characteristics of the particle. The black line indicates the boundary between passing and trapped particles (at $\epsilon=r / R_{0}$ $=0.17$ ), the dashed line corresponds to $D=0.1 \mathrm{~m}^{2} \mathrm{~s}^{-1}$, and the dashed dotted line indicates the critical energy boundary $\left(E_{c}=300 \mathrm{keV}\right)$. It is clear how $D_{\text {an }}>D_{\text {neo }}$ is observed for a large region in phase space.

analytical scalings of Ref. 31, and draw more quantitative conclusions, we represent the data as a function of energy only for a fixed pitch angle (Fig. 7). Here we consider two slices of the function $D_{v}$ at different energies while keeping $\mu B_{0} / E$ fixed. One slice has been performed at $\mu B_{0} / E$ $=0.05$ (small Larmor radius, deeply passing particles) and the other at $\mu B_{0} / E=0.9$ (deeply trapped particles). The trapped/passing boundary is located at $\mu B_{0} / E \simeq 0.7$. We note that these pitch angles are characteristic of tangentially injected NBI distributions and ICRH distributions, respectively. For passing ions, a $D_{v} \simeq E^{-1}$ decay is expected. ${ }^{29-31}$ For deeply trapped particles, i.e., ICRH heated ions, a faster energy decay $D_{v} \simeq E^{-3 / 2}$ has been proposed in Ref. 31. Similar scalings have been found in the results shown in Fig. 7, although some discrepancies can be found at energies exceeding $1 \mathrm{MeV}$. A possible explanation for this disagreement resides in the definition of $D_{v}$ given by Eq. (17), where a normalization with respect to the unperturbed $f_{0}$ is present. Having energies above $1 \mathrm{MeV}$ on the tail of the Maxwellian
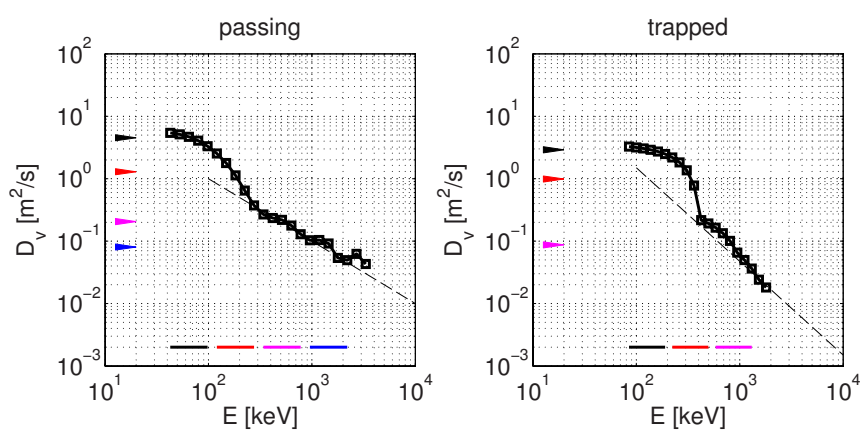

FIG. 7. (Color online) Anomalous transport of deeply passing $\left(\mu B_{0} / E\right.$ $=0.05)$ and deeply trapped $\left(\mu B_{0} / E=0.9\right)$ particles as a function of energy. For both classes of particles, an enhancement of radial transport is observed for supracritical energies, namely, $300 \mathrm{keV}$. The colored arrows correspond to an average of $D_{v}$ over the energy range specified by the solid lines characterized by the same color at the bottom of the figure. The dashed lines represent the high energy decay proposed in Ref. 31, namely, $E^{-1}$ for passing and $E^{-3 / 2}$ for trapped particles. 
$f_{0}$ leads to possible truncation errors in the procedure. Nevertheless, the neoclassical limit is exceeded at energies below $700 \mathrm{keV}$ for trapped and below $1 \mathrm{MeV}$ for passing particles. These results suggest that ITER, NBI, and ICRH heated particles can be subject to a strong anomalous transport with the result of drifting far from the deposition layer before slowing down. As the isotropic alpha particles can be characterized by both passing and trapped orbits, a reduction in the plasma self-heating is also to be expected.

Trapped and passing energetic ions are characterized by very different orbits. As turbulent fields are present only in a narrow region at midradius, some particles could still be able to slow down classically once they have been diffused outside the turbulent layer. This is particularly true for passing ions, whose unperturbed trajectories are not particularly wide in the poloidal cross section. On the other hand, highly energetic trapped ions, whose orbits are distinguished by a large width, could be promptly expelled, or enter regions where ripple losses become important. Calculations of heat loads on material walls, and also heating efficiency in general, should include the anomalous transport of energetic ions considered in this paper.

\section{FINITE BETA EFFECTS ON THE TRANSPORT OF SUPRATHERMAL IONS}

In Sec. I we pointed out that the simulations performed throughout the paper only consider the $E \times B$ component of $\delta \mathbf{v}$ in Eq. (1). Finite $\beta$ effects nevertheless play an important role in determining the time evolution of the perturbed fields and, consequently, of the distribution function of the passive tracers. We now assess the difference between a system characterized by $\beta \simeq 0$ (purely electrostatic perturbations) and the simulations presented in Sec. IV where $\beta=0.6 \% \equiv \beta^{\text {high }}$. This particular value has been chosen as it represents half the value required for the onset of kinetic ballooning modes, as reported in Sec. III. A numerical investigation of a system characterized by the same parameters introduced in Sec. III for ITER has been carried out, the only difference being in the choice of $\beta=10^{-4} \equiv \beta^{\text {low }}$. In the linear phase, the growth rate of the most unstable mode increases from $\gamma$ $=0.12 c_{s} / R_{0}$ for $\beta^{\text {high }}$ to $\gamma=0.35 c_{s} / R_{0}$ for $\beta^{\text {low }}$. These results agree with the findings of Refs. 35 and 38-40, where it was observed that the increase in $\beta$ has a stabilizing effect on gyrokinetic instabilities. The nonlinear evolution of the simulation reflects the larger degree of instability of the system when $\beta$ is small, as the diffusivity of the population at $T$ $=30 T_{e}$ is now $D=5 \mathrm{~m}^{2} \mathrm{~s}^{-1}$, a value considerably larger than $D=0.75 \mathrm{~m}^{2} \mathrm{~s}^{-1}$, found in Sec. III when employing a finite $\beta$. A similar enhancement is observed in the alpha particle heat diffusivity that increases from $\chi_{\text {eff }} \simeq 3 \mathrm{~m}^{2} \mathrm{~s}^{-1}$ to $\chi_{\text {eff }}$ $\simeq 21 \mathrm{~m}^{2} \mathrm{~s}^{-1}$ when decreasing $\beta$. When exploring the velocity space dependence of the particle diffusivity, as in Sec. IV, a large increase in the transport is again observable (Fig. 8). It is thus clear that finite $\beta$ effects must be retained in the simulation of a burning plasma. Finally, it should be mentioned that magnetic effects on the perturbed velocity, not considered so far, enter the definition of a particle flux as
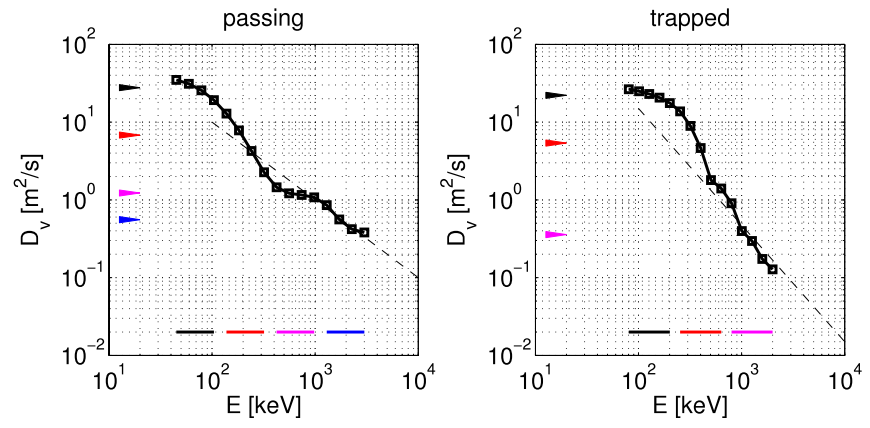

FIG. 8. (Color online) Anomalous transport of deeply passing $\left(\mu B_{0} / E\right.$ $=0.05)$ and deeply trapped $\left(\mu B_{0} / E=0.9\right)$ particles as a function of energy in the presence of electrostatic turbulent fields. The transport of particles in a low $\beta$ system is considerably larger than that of a finite $\beta$ simulation (see Fig. 7 for comparison). The dashed lines represent the high energy decay proposed in Ref. 31, namely, $E^{-1}$ for passing and $E^{-3 / 2}$ for trapped particles. For this particular case, the scaling for deeply passing particles is slightly larger than the one predicted by the theory. At the same time, the diffusion coefficient for deeply trapped particles appears to decay faster than predicted.

$$
\boldsymbol{\Gamma}=\int d \mathbf{v} \delta f\left(\delta \mathbf{v}_{E \times B}+\delta \mathbf{v}_{A_{\|}}\right) .
$$

The term proportional to $\delta \mathbf{v}_{A_{\|}}$partially compensates the decrease in transport with increasing $\beta$, as the intensity of the perturbed magnetic potential is enhanced. This effect is especially important for high energy ions, as the perturbed magnetic fields induce drifts proportional to the particle velocity (see, e.g., Ref. 41). This subject will be thoroughly treated in a future paper.

\section{CONCLUSIONS}

We have studied the transport of energetic particles in the presence of small-scale turbulent fields generated by a background plasma of ions and electrons. Nonlinear gyrokinetic simulations of an ITER steady-state scenario have been performed using the GENE code employing the $s-\alpha$ equilibrium. Due to their low concentration, fast ion populations have been considered as passive tracers, not influencing the time evolution of the turbulent fields. ITER-relevant parameters and a velocity space dependent diffusivity are introduced, shedding light on the possibility that anomalous transport could enhance fast ion redistribution in fusion plasmas.

Two passive populations, describing helium ash impurities and alpha particles, have been introduced in the calculation. The results show that burning plasma regimes can be affected by a low temperature pinch, potentially leading to impurity retention, as well as by a non-negligible transport of populations whose energies largely exceed the plasma temperature. The latter result, in particular, is surprising, since gyro- and orbit-averaging effects are normally expected to suppress turbulent transport whenever a population is characterized by a large number of energetic ions.

In order to verify these conclusions, an ITER steadystate scenario discharge has been modeled by choosing density and temperature profiles from ASTRA calculations. ${ }^{33,34}$ The GENE code employed for simulating this scenario assumes Maxwellian stationary functions. By separating the low and high energy parts of the slowing down distribution 
of fusion born products, two Maxwellian populations have been constructed, and nonlinear simulations performed. The results show how the low temperature pinch previously observed is compensated by a strong $\nabla n$-driven outward flux. However, a non-negligible diffusivity of $D \geq 0.75 \mathrm{~m}^{2} \mathrm{~s}^{-1}$ has again been obtained when the temperature of the (high energy) Maxwellian population reaches values of the order of $T_{\alpha}=450 \mathrm{keV}$.

As a model based on the collective transport of a population does not distinguish between the thermalized bulk particles and those of high energy but low concentration, a velocity space dependent diffusivity has been introduced. We have set $0.1 \mathrm{~m}^{2} \mathrm{~s}^{-1}$ as an upper limit for the collisional diffusivity of ions above $E_{c}=300 \mathrm{keV}$ and demonstrated how enhanced transport of energetic particles is present over a wide range of energies. Passing particles are the most affected by the presence of turbulent fields, as ions up to 1 $\mathrm{MeV}$ experience diffusivities larger than $0.1 \mathrm{~m}^{2} \mathrm{~s}^{-1}$. A smaller amount of transport has been found for trapped particles, as gyroaveraging effects give rise to the faster decay formulated in Ref. 31. Their diffusivity has been found to be important only in the energy range of 300-700 keV. Our findings have been shown to be in qualitative agreement with those presented in Refs. 29, 31, and 41, and quantitative discrepancies are due to the difference in the parameters chosen.

This study indicates that burning plasma operations can be influenced by the presence of small-scale turbulent fields. As the radial extension of these fields is usually small, energetic particles can still transfer their energy once diffused outside the turbulent layer. Transport models should nevertheless account for possible changes in the heat deposition profiles. At the same time, small fractions of energetic particles driven toward the low field side of the plasma can be expelled due to first orbit or ripple losses. As a consequence, enhanced heat load on plasma facing materials can be expected, whose intensity needs to be evaluated in detail.

\section{ACKNOWLEDGMENTS}

This work was partly supported by the Swiss National Science Foundation. The simulations have been run on the Pleiades2 cluster at the Ecole Polytechnique Fédérale de Lausanne. We gratefully acknowledge fruitful discussions with X. Lapillonne, M. J. Pueschel, and T. Görler.

\footnotetext{
${ }^{1}$ R. Aymar, V. A. Chuyanov, M. Huguet, Y. Shimomura, ITER Joint Central Team, and ITER Home Teams, Nucl. Fusion 41, 1301 (2001).

${ }^{2}$ A. Fasoli, C. Gormenzano, H. Berk, B. Breizman, S. Briguglio, D. Darrow, N. Gorelenkov, W. Heidbrink, A. Jaun, S. Konovalov, R. Nazikian, J.-M. Noterdaeme, S. Sharapov, K. Shinohara, D. Testa, K. Tobita, Y. Todo, G. Vlad, and F. Zonca, Nucl. Fusion 47, S264 (2007).

${ }^{3}$ R. B. White and H. E. Mynick, Phys. Fluids B 1, 980 (1989).

${ }^{4}$ J. R. Myra, P. J. Catto, H. E. Mynick, and R. E. Duvall, Phys. Fluids B 5, 1160 (1993).

${ }^{5}$ G. Manfredi and R. O. Dendy, Phys. Rev. Lett. 76, 4360 (1996).

${ }^{6}$ R. J. Hawryluk and the TFTR team, Phys. Plasmas 5, 1577 (1998).

${ }^{7}$ W. W. Heidbrink, C. W. Barnes, G. W. Hammett, Y. Kusama, S. D. Scott, M. C. Zarnstorff, L. C. Johnson, D. McCune, S. S. Medley, H. K. Park, A. L. Roquemore, J. D. Strachan, and G. Taylor, Phys. Fluids B 3, 3167 (1991).

${ }^{8}$ E. Ruskov, W. W. Heidbrink, and R. V. Budny, Nucl. Fusion 12, 2219 (1991).
}

${ }^{9}$ E. Ruskov, W. W. Heidbrink, and R. V. Budny, Nucl. Fusion 35, 1099 (1995).

${ }^{10}$ A. Herrmann and O. Gruber, Fusion Sci. Technol. 44, 569 (2003).

${ }^{11}$ S. Günter, G. Conway, S. da Graça, H.-U. Fahrbach, C. Forest, M. Garcia Muñoz, T. Hauff, J. Hobirk, V. Igochine, F. Jenko, K. Lackner, P. Lauber, P. McCarthy, M. Maraschek, P. Martin, E. Poli, K. Sassenberg, E. Strumberger, G. Tardini, E. Wolfrum, H. Zohm, and ASDEX Upgrade Team, Nucl. Fusion 47, 920 (2007).

${ }^{12}$ M. Keilhacker, A. Gibson, C. Gormezano, and P. H. Rebut, Nucl. Fusion 41, 1925 (2001)

${ }^{13}$ Y. F. Baranov, I. Jenkins, B. Alper, C. D. Challis, S. Conroy, V. Kiptily, J. Ongena, S. Popovichev, P. Smeulders, E. Surrey, K.-D. Zastrow, and JET EFDA contributors, Plasma Phys. Controlled Fusion 51, 044004 (2009).

${ }^{14}$ A. Sykes, R. J. Akers, L. C. Appel, E. R. Arends, P. G. Carolan, N. J. Conway, G. F. Counsell, G. Cunningham, A. Dnestrovskij, Y. N. Dnestrovskij, A. R. Field, S. J. Fielding, M. P. Gryaznevich, S. Korsholm, E. Laird, R. Martin, M. P. S. Nightingale, C. M. Roach, M. R. Tournianski, M. J. Walsh, C. D. Warrick, H. R. Wilson, S. You, MAST Team, and NBI Team, Nucl. Fusion 41, 1423 (2001).

${ }^{15}$ M. Turnyanskiy, D. L. Keeling, R. J. Akers, G. Cunningham, N. J. Conway, H. Meyer, C. A. Michael, and S. D. Pinches, Nucl. Fusion 49, 065002 (2009).

${ }^{16}$ J. L. Luxon, Nucl. Fusion 42, 614 (2002).

${ }^{17}$ M. Murakami, J. M. Park, C. C. Petty, T. C. Luce, W. W. Heidbrink, T. H. Osborne, R. Prater, M. R. Wade, P. M. Anderson, M. E. Austin, N. H. Brooks, R. V. Budny, C. D. Challis, J. C. DeBoo, J. S. de Grassie, J. R. Ferron, P. Gohil, J. Hobirk, C. T. Holcomb, E. M. Hollmann, R. M. Hong, A. W. Hyatt, J. Lohr, M. J. Lanctot, M. A. Makowski, D. C. McCune, P. A. Politzer, J. T. Scoville, H. E. St. John, T. Suzuki, T. S. Taylor, W. P. West, E. A. Unterberg, M. A. Van Zeeland, and J. H. Yu, Nucl. Fusion 49, 065031 (2009)

${ }^{18}$ T. Hauff and F. Jenko, Phys. Plasmas 13, 102309 (2006).

${ }^{19}$ T. Hauff and F. Jenko, Phys. Plasmas 14, 092301 (2007).

${ }^{20}$ J. Candy and R. Waltz, Phys. Rev. Lett. 91, 045001 (2003).

${ }^{21}$ C. Estrada-Mila, J. Candy, and R. E. Waltz, Phys. Plasmas 13, 112303 (2006).

${ }^{22}$ C. Angioni and A. G. Peeters, Phys. Plasmas 15, 052307 (2008).

${ }^{23}$ C. Angioni, A. G. Peeters, G. V. Pereverzev, A. Bottino, J. Candy, R. Dux,

E. Fable, T. Hein, and R. E. Waltz, Nucl. Fusion 49, 055013 (2009).

${ }^{24}$ M. Kotschenreuther, G. Rewoldt, and W. M. Tang, Comput. Phys. Commun. 88, 128 (1995).

${ }^{25}$ W. Dorland, F. Jenko, M. Kotschenreuther, and B. N. Rogers, Phys. Rev. Lett. 85, 5579 (2000).

${ }^{26}$ A. G. Peeters and D. Strintzi, Phys. Plasmas 11, 3748 (2004).

${ }^{27}$ F. Jenko, W. Dorland, M. Kotschenreuther, and B. N. Rogers, Phys. Plasmas 7, 1904 (2000).

${ }^{28}$ T. Dannert and F. Jenko, Phys. Plasmas 12, 072309 (2005).

${ }^{29}$ T. Dannert, S. Günter, T. Hauff, F. Jenko, X. Lapillonne, and P. Lauber, Phys. Plasmas 15, 062508 (2008).

${ }^{30}$ W. Zhang, Z. Lin, and L. Chen, Phys. Rev. Lett. 101, 095001 (2008).

${ }^{31}$ T. Hauff and F. Jenko, Phys. Plasmas 15, 112307 (2008).

${ }^{32}$ J. W. Connor, R. J. Hastie, and J. B. Taylor, Phys. Rev. Lett. 40, 396 (1978).

${ }^{33}$ C. Gormezano, A. C. C. Sips, T. C. Luce, S. Ide, A. Becoulet, X. Litaudon, A. Isayama, J. Hobirk, M. R. Wade, T. Oikawa, R. Prater, A. Zvonkov, B. Lloyd, T. Suzuki, E. Barbato, P. Bonoli, C. K. Phillips, V. Vdovin, E. Joffrin, T. Casper, J. Ferron, D. Mazon, D. Moreau, R. Bundy, C. Kessel, A. Fukuyama, N. Hayashi, F. Imbeaux, M. Murakami, A. R. Polevoi, and H. E. St. John, Nucl. Fusion 47, S285 (2007).

${ }^{34}$ C. Zucca, O. Sauter, M. A. Henderson, E. Fable, D. Farina, A. Polevoi, G. Ramponi, G. Saibene, and H. Zohm, AIP Conf. Proc. 1069, 361 (2008).

${ }^{35}$ M. J. Pueschel, M. Kammerer, and F. Jenko, Phys. Plasmas 15, 102310 (2008).

${ }^{36}$ J. D. Gaffey, Jr., J. Plasma Phys. 16, 149 (1976)

${ }^{37}$ ITER Physics Expert Group on Energetic Particles, Heating and Current Drive and ITER Physics Basis Editors, Nucl. Fusion 39, 2471 (1999).

${ }^{38}$ F. Jenko and W. Dorland, Plasma Phys. Controlled Fusion 43, A141 (2001).

${ }^{39}$ J. Candy, Phys. Plasmas 12, 072307 (2005).

${ }^{40}$ Y. Chen, S. E. Parker, B. I. Cohen, A. M. Dimits, W. M. Nevins, D. Shumaker, V. K. Decyk, and J. N. Leboeuf, Nucl. Fusion 43, 1121 (2003).

${ }^{41}$ T. Hauff, M. J. Pueschel, T. Dannert, and F. Jenko, Phys. Rev. Lett. 102, 075004 (2009). 